\title{
Potenciais interações entre medicamentos usados na síndrome metabólica
}

\author{
Potential interactions between drugs used in metabolic syndrome
}

Vanessa Adelina Casali Bandeira', Karla Renata de Oliveira ${ }^{2}$

${ }^{1}$ Acadêmica do Curso de Graduação em Farmácia do Departamento de Ciências da Vida - DCVida da Universidade Regional do Noroeste do Estado do Rio Grande do Sul (UNIJUÍ), Ijuí, RS; '2Farmacêutica, mestre em Ciências Biológicas/Bioquímica; Docente do DCVida da UNIJUÍ, ljuí, RS.

\section{RESUMO}

Objetivos: Identificar os medicamentos indicados para o controle das doenças crônicas não transmissíveis que constituem a síndrome metabólica, incluídos na Relação Nacional de Medicamentos Essenciais, e as potenciais interações medicamentosas positivas e negativas entre os mesmos, classificando-as de acordo com a gravidade.

Métodos: Foram identificados os medicamentos utilizados para o controle das doenças crônicas não transmissíveis por meio da I Diretriz Brasileira de Diagnóstico e Tratamento da Síndrome Metabólica e das diretrizes específicas de cada doença que constitui a síndrome metabólica. Dentre estes, foram selecionados os elencados na Relação Nacional de Medicamentos Essenciais. Na segunda parte do estudo, através de uma pesquisa na literatura identificaram-se as potenciais interações medicamentosas, as quais foram classificadas em positivas e negativas, assim como de acordo com a sua gravidade.

Resultados: Foram identificados na Relação Nacional de Medicamentos Essenciais 19 medicamentos, cada um apresentou pelo menos uma interação medicamentosa potencial, totalizando 89 interações. Dentre estas, 20,22\% foram classificadas como positivas, sendo representadas principalmente pela associação de anti-hipertensivos; e 79,78\% foram consideradas negativas, das quais 14,09\% apresentam gravidade maior.

Conclusões: O tratamento da síndrome metabólica é complexo por requerer a associação de vários medicamentos, o que aumenta os riscos de interações medicamentosas e de efeitos adversos. O presente estudo identificou um grande número de potenciais interações medicamentosas entre esses medicamentos, o que pode reduzir ou potencializar a eficácia dos mesmos e expor o usuário a riscos.

DESCRITORES: DIABETES MELLITUS; DISLIPIDEMIAS; HIPERTENSÃO; INTERAÇ̃̃O MEDICAMENTOSA; OBESIDADE; SÍNDROME X METABÓLICA; TRATAMENTO MEDICAMENTOSO.

\section{ABSTRACT}

Aims: To identify the drugs indicated for the control of chronic noncommunicable diseases that constitute the metabolic syndrome, included in the National List of Essential Medicines, and the potential positive and negative drug interactions between them, classifying them according to severity.

Methods: The drugs used for the control of chronic noncommunicable diseases through Brazilian Guideline for Diagnosis and Treatment of Metabolic Syndrome, as well as specific guidelines for each disease that constitute metabolic syndrome were identified. Among these, we selected those listed in the National List of Essential Medicines. In the second part of the study, through a literature search, the potential drug interactions were identified, and were classified as positive or negative and according to their severity.

Results: Nineteen drugs were identified in the National List of Essential Medicines, each of which had at least one potential drug interaction, totaling 89 potential interactions. Among these, $20.22 \%$ were classified as positive, these being mainly represented by the association of antihypertensive drugs; and $79.78 \%$ were considered negative, of which $14.09 \%$ had great severity.

Conclusions: The treatment of metabolic syndrome is complex because it requires the combination of several drugs, which increases the risk of drug interactions and adverse effects. This study identified a number of potential drug interactions between these drugs, which can reduce or enhance their effectiveness and expose the patient to risks.

KEY WORDS: DIABETES MELLITUS; DYSLIPIDEMIAS; HYPERTENSION; DRUG INTERACTION; OBESITY; METABOLIC SYNDROME X; DRUG THERAPY. 


\section{INTRODUÇÃO}

A transição demográfica e avanços na área da saúde determinaram o aumento da expectativa de vida da população, que associados a mudanças nos hábitos de vida, predispõem a doenças crônicas não transmissíveis (DCNT), como hipertensão arterial sistêmica (HAS), diabetes mellitus (DM) e doenças cardiovasculares (DCV), sendo estas últimas as principais causas de morbidade e mortalidade no mundo., ${ }^{1,2}$

De acordo com a Organização Mundial da Saúde $(\mathrm{OMS})^{3}$ as DCNT estão aumentando em ritmo acelerado mundialmente. Essas doenças demandam cuidados contínuos, por período prolongado, o que afeta tanto os doentes quanto os serviços de saúde. ${ }^{3}$ De acordo com a Organização Pan Americana de Saúde (OPAS), ${ }^{4}$ no ano de 2010 o número de óbitos por DM a cada 100 mil habitantes foi de 22 na América do Norte, 36 na América Latina e 30 no Brasil. Em relação à população brasileira, $9,9 \%$ é diabética e $23,3 \%$ hipertensa. ${ }^{5}$ Salienta-se que a HAS representa o fator de risco mais importante para a manifestação de DCV, sendo que o acidente vascular cerebral e o infarto do miocárdio são as maiores causas isoladas de morte no Brasil. ${ }^{6}$

Uma DCNT não pode ser avaliada isoladamente, principalmente na presença de outras condições crônicas, pois cada uma das DCNT está associada a um risco cardiovascular individual, que aumenta exponencialmente quando se apresentam de forma associada. ${ }^{7}$ Nesse contexto, surgiu o conceito de Síndrome Metabólica (SM), um transtorno metabólico complexo, representado por um conjunto de fatores de risco cardiovascular. ${ }^{8}$

No Brasil, a I Diretriz Brasileira de Diagnóstico e Tratamento da Síndrome Metabólica (I-DBSM) ${ }^{8}$ apresenta a definição de SM baseada no Third Report of the National Cholesterol Education Program Expert Panel on Detection, Evaluation, and Treatment of High Blood Cholesterol in Adults (NCEP ATP III). ${ }^{9}$ Assim, a definição para o diagnóstico da SM compreende a presença de três das seguintes condições: obesidade verificada por meio da circunferência abdominal; triglicerídeos $\geq 150 \mathrm{mg} / \mathrm{dL}$; colesterol ligado a lipoproteínas de alta densidade (HDL-colesterol) $<40 \mathrm{mg} / \mathrm{dL}$ em homens e $<50 \mathrm{mg} / \mathrm{dL}$ em mulheres e/ ou uso de medicação hipolipemiante; pressão arterial sistêmica $\geq 130 \mathrm{mmHg}$ (sistólica) ou $\geq 85 \mathrm{mmHg}$ (diastólica) e/ou uso de medicação anti-hipertensiva; e glicemia de jejum $\geq 110 \mathrm{mg} / \mathrm{dL}{ }^{8}$

A SM é extremamente relevante para a condição de saúde dos indivíduos e seu tratamento é imprescindível, visando controlar as doenças existentes e o risco cardiovascular inerente. ${ }^{8}$ Destaca-se, ainda, que por se tratar de uma condição complexa requer o uso concomitante de dois ou mais medicamentos. E, segundo Secoli, ${ }^{10}$ o número de medicamentos em uso está relacionado ao aumento na ocorrência de reações adversas e interações medicamentosas (IM). Nesse contexto, a polifarmácia, caracterizada pelo uso concomitante de vários medicamentos, torna-se necessária para o tratamento de doenças coexistentes, no controle de reações indesejadas, ou para potencializar o efeito farmacológico em condições refratárias e pouco responsivas. No entanto, a combinação de fármacos pode reduzir a sua eficácia e promover reações adversas. ${ }^{11}$

Quando dois ou mais medicamentos são administrados concomitantemente, eles podem agir independentemente ou interagir, o que pode modificar sua ação farmacológica, caracterizando uma IM. ${ }^{12}$ Essa interação pode desencadear efeitos sinérgicos ou antagônicos, entre os quais se incluem interações contraindicadas, que podem reduzir a ação dos fármacos ou aumentar sua toxicidade. ${ }^{12}$ Ainda, as IM podem se apresentar com caráter potencial, ou seja, existe a possibilidade de um medicamento alterar o efeito do outro administrado concomitantemente, no entanto isso dependerá de um conjunto de fatores. ${ }^{13}$

Neste sentido, o presente estudo propôs-se a identificar os medicamentos indicados para o controle das DCNT que constituem a SM incluídos na Relação Nacional de Medicamentos Essenciais (RENAME) e pesquisar as potenciais IM entre os mesmos, classificando-as de acordo com a sua gravidade.

\section{MÉTODOS}

Este estudo constitui-se em uma pesquisa exploratória dos medicamentos utilizados no controle da SM, seguida por uma revisão bibliográfica que buscou identificar as potenciais IM entre os mesmos. Para a identificação dos medicamentos indicados para o controle das DCNT que constituem a SM foram considerados os elencados na I-DBSM. ${ }^{8}$ Também foram consultadas as VI Diretrizes Brasileiras de Hipertensão, Diretrizes da Sociedade Brasileira de Diabetes, Diretrizes Brasileiras de Obesidade e IV Diretriz Brasileira sobre Dislipidemias e Prevenção da Aterosclerose. ${ }^{6,14-16}$ Entre os medicamentos identificados, foram selecionados para o presente estudo aqueles que compõem a RENAME, ${ }^{17}$ sétima edição, por se constituir na última versão impressa, lançada por meio de processo de análise comparativa, 
com base em evidência clínica, justificando exclusões e inclusões, indicações e restrições de uso. Além disso, constitui-se na última edição na qual a publicação foi acompanhada pelo Formulário Terapêutico Nacional $(\text { FTN })^{18}$ que contém as informações científicas dos medicamentos presentes na RENAME. Os medicamentos selecionados foram classificados no primeiro e quarto nível do sistema de classificação Anatomical Therapeutic Chemical (ATC). ${ }^{19}$

A pesquisa bibliográfica foi realizada visando identificar as potenciais IM entre os medicamentos selecionados, no período de agosto a outubro de 2012, através das bases de dados Drugs.com ${ }^{20}$ e Micromedex ${ }^{21}$ e livros publicados entre 2006 e 2012, em língua portuguesa e inglesa. As IM foram identificadas ainda de acordo com Tatro ${ }^{22}$ e FTN ${ }^{18}$ A classificação das IM segundo a relevância clínica consistiu em: menor (avaliar e considerar medicamentos alternativos, tomar medidas para contornar o risco de interação e/ou instituir um plano de monitoramento); moderada (devido ao risco dessa interação recomenda-se usar apenas em circunstâncias especiais); e maior (com alta significância clínica, pois o risco dessa interação supera o benefício)..$^{20,21}$

As IM foram classificadas, ainda, em positivas ou negativas. Foram consideradas positivas aquelas que oferecem benefício e apresentam eficácia comprovada de acordo com as diretrizes supracitadas, e negativas as que não oferecem benefícios, expõem os usuários a riscos e não apresentam eficácia comprovada.

\section{RESULTADOS}

\section{Medicamentos disponíveis na RENAME}

Foram identificados 93 princípios ativos indicados para o controle das DCNT que constituem a SM, dos quais $19(20,43 \%)$ estão presentes na RENAME, ${ }^{17}$ apresentados com a respectiva classe terapêutica e classificação ATC no primeiro nível (grupo anatômico principal) e quarto nível (subgrupo farmacológico) na Tabela 1.

Treze medicamentos $(68,42 \%)$ são indicados para o controle da HAS, dentre os quais se encontram anlodipino, atenolol, captopril, carvedilol, enalapril, espironolactona, furosemida, hidralazina, hidroclorotiazida, losartana, metoprolol, propranolol e verapamil. O nifedipino, medicamento com indicação anti-hipertensiva, foi excluído do presente estudo por estar presente na RENAME ${ }^{17}$ para uso restrito como tocolítico. ${ }^{17,18}$

Entre os medicamentos indicados para o controle do DM, três $(15,79 \%)$ estão presentes na RENAME ${ }^{17}$ glibenclamida, gliclazida e metformina. Dois $(10,53 \%)$ são hipolipemiantes, representados pelo fenofibrato e sinvastatina, e um $(5,26 \%)$ é indicado para a obesidade.

Tabela 1. Medicamentos identificados na Relação Nacional de Medicamentos Essenciais (RENAME) indicados para o controle das doenças crônicas não transmissíveis que constituem a síndrome metabólica, classificados de acordo com o primeiro e quarto nível da Anatomical Therapeutic Chemical ATC/DDD Index 2014

\begin{tabular}{|c|c|c|}
\hline 1o Nível & 4o Nível & Fármacos \\
\hline \multirow[t]{2}{*}{ A - Trato alimentar e metabolismo } & A10BB - Sulfonamidas, derivados da uréia & Glibenclamida e Gliclazida \\
\hline & A10BA - Biguanidas & Metformina \\
\hline \multirow[t]{13}{*}{ C - Sistema Cardiovascular } & C02DB - Derivados hidrazinoftalazina & Hidralazina \\
\hline & C03AA - Tiazidas, monodroga & Hidroclorotiazida \\
\hline & C03CA - Sulfonamidas, monodroga & Furosemida \\
\hline & C03DA - Antagonistas da aldosterona & Espironolactona \\
\hline & C07AA - Betabloqueadores não seletivos & Propranolol \\
\hline & C07AB - Agentes betabloqueadores, seletivos & Atenolol e Metoprolol \\
\hline & C07AG - Agentes bloqueadores alfa e beta & Carvedilol \\
\hline & C08CA - Derivados da dihidropiridina & Anlodipino \\
\hline & C08DA - Derivados da fenilaquilamina & Verapamil \\
\hline & C09AA - Inibidores da enzima conversora da angiotensina, monodroga & Captopril e Enalapril \\
\hline & C09CA - Antagonistas da angiotensina II, monodroga & Losartana \\
\hline & C10AA - Inibidores da HMG CoA redutase & Sinvastatina \\
\hline & C10BA - Fibratos & Fenofibrato \\
\hline $\mathrm{N}$ - Sistema Nervoso & N06AB - Inibidores seletivos da recaptação de serotonina & Fluoxetina \\
\hline
\end{tabular}

HMG CoA: 3-hidroxi-3-metil-glutaril-Coenzima A. 
Este último medicamento, a fluoxetina, é utilizado no tratamento da depressão e não tem indicação formal para o tratamento da obesidade; no entanto, nas Diretrizes Brasileiras de Obesidade ${ }^{15}$ está indicado para esse fim.

\section{Potenciais interações medicamentosas}

Entre os 19 medicamentos presentes na RENAME ${ }^{17}$ foram identificadas 89 potenciais IM, sendo 18 $(20,22 \%)$ positivas e $71(79,78 \%)$ negativas. Nas interações positivas predominam as associações entre duas classes distintas de anti-hipertensivos, assim como associação entre diuréticos (hidroclorotiazida e furosemida), diuréticos e losartana, hidroclorotiazida e anlodipino, inibidores da enzima conversora da angiotensina (IECA) e anlodipino ou diuréticos ou verapamil, e a associação entre hidralazina e betabloqueadores. Ainda, foi observada uma IM positiva quando sulfoniluréias (glibenclamida ou gliclazida) são associadas a metformina para o controle do DM.

Em relação às IM negativas duas $(2,81 \%)$ foram classificadas como menores, $59(83,10 \%)$ moderadas e $10(14,09 \%)$ maiores (Tabela 2).

Entre os medicamentos indicados como antihipertensivos incluídos na RENAME $^{17}$ estão os diuréticos, como hidroclorotiazida e furosemida, que podem exercer efeito hipotensor aditivo, podendo, entretanto, resultar em intensa diurese e anormalidades eletrolíticas. ${ }^{18}$ Ainda, diuréticos são frequentemente associados a outros anti-hipertensivos, como IECA e losartana,${ }^{8}$ a fim de promover adição do efeito antihipertensivo, sendo esta uma interação positiva, pois se busca uma melhor resposta terapêutica; no entanto, é necessário monitorar sintomas e sinais relacionados à ocorrência de hipotensão. ${ }^{8,18,22}$ Além disso, os diuréticos interagem com medicamentos hipoglicemiantes, reduzindo seu efeito ${ }^{20,21}$ enquanto a associação com fluoxetina aumenta o risco de hiponatremia. ${ }^{20} \mathrm{~A}$ depuração plasmática da furosemida pode ser aumentada quando associada a hidralazina. ${ }^{20}$ Espironolactona pode interagir com antagonistas do receptor de angiotensina II (losartana) e IECA (captopril e enalapril), e a interação com ambas as classes pode resultar em elevação na concentração sérica do potássio, sendo de alto risco, principalmente para diabéticos e portadores de insuficiência renal. . $^{180-22}$

Os betabloqueadores, classe de anti-hipertensivos com o maior número de representantes (atenolol, carvedilol, metoprolol e propranolol) na RENAME ${ }^{17}$ apresenta potencial IM com os medicamentos de primeira escolha para o DM, podendo interferir no efeito hipoglicemiante da glibenclamida e da metformina, e causar hiper ou hipoglicemia; quando necessário o uso da associação, é indispensável o monitoramento dos níveis glicêmicos..$^{21,22}$

Tabela 2. Potenciais interações medicamentosas negativas e respectivas classificações, entre os medicamentos (fármacos da primeira com os da segunda coluna) presentes da Relação Nacional de Medicamentos Essenciais (RENAME) indicados para o controle dos componentes da síndrome metabólica

\begin{tabular}{|c|c|c|}
\hline Fármacos & Fármacos & $\begin{array}{l}\text { Classificação da } \\
\text { interação }\end{array}$ \\
\hline Hidroclorotiazida & - Atenolol, Carvedilol, Metoprolol, Propranolol, Glibenclamida, Gliclazida, Metformina e Fluoxetina & Moderada \\
\hline Furosemida & $\begin{array}{l}\text { - Hidralazina } \\
\text { - Atenolol, Carvedilol, metoprolol, Propranolol, Glibenclamida, Gliclazida, Metformina e Fluoxetina }\end{array}$ & $\begin{array}{l}\text { Menor } \\
\text { Moderada }\end{array}$ \\
\hline Espironolactona & $\begin{array}{l}\text { - Atenolol, Carvedilol, metoprolol, Propranolol, Metformina e Fluoxetina } \\
\text { - Captopril, Enalapril e Losartana }\end{array}$ & $\begin{array}{l}\text { Moderada } \\
\text { Maior }\end{array}$ \\
\hline $\begin{array}{l}\text { Atenolol, Carvedilol, } \\
\text { Metoprolol e Propranolol }\end{array}$ & $\begin{array}{l}\text { - Anlodipino, Glibenclamida, Gliclazida, Metformina e Fluoxetina } \\
\text { - Verapamil }\end{array}$ & $\begin{array}{l}\text { Moderada } \\
\text { Maior }\end{array}$ \\
\hline Hidralazina & - Sinvastatina & Moderada \\
\hline Verapamil & $\begin{array}{l}\text { - Glibenclamida } \\
\text { - Fluoxetina } \\
\text { - Sinvastatina }\end{array}$ & $\begin{array}{l}\text { Menor } \\
\text { Moderada } \\
\text { Maior }\end{array}$ \\
\hline Anlodipino & $\begin{array}{l}\text { - Fluoxetina } \\
\text { - Sinvastatina }\end{array}$ & $\begin{array}{l}\text { Moderada } \\
\text { Maior }\end{array}$ \\
\hline Captopril e Enalapril & $\begin{array}{l}\text { - Losartana*, Glibenclamida, Gliclazida, Metformina } \\
\text { - Losartana* }\end{array}$ & $\begin{array}{l}\text { Moderada } \\
\text { Maior }\end{array}$ \\
\hline Glibenclamida e Gliclazida & - Fenofibrato, Sinvastatina e Fluoxetina & Moderada \\
\hline Fenofibrato & - Sinvastatina & Maior \\
\hline
\end{tabular}

* A interação entre captopril ou enalapril com losartana foi a única em que a classificação da interação variou conforme a referência bibliográfica, considerada como moderada por Tatro ${ }^{20}$ e maior por Micromedex. ${ }^{21}$ 
Destaca-se que os betabloqueadores são indicados como primeira opção no tratamento da HAS, no entanto, podem induzir ao aumento de peso e à intolerância à glicose, e com isso aumentar o risco para o desenvolvimento de DM, hipertrigliceridemia e redução do HDL-colesterol. Esses riscos são potencializados se esses medicamentos forem combinados a um diurético. ${ }^{6,8}$ Dessa forma, não são recomendados no tratamento da SM, principalmente em diabéticos em uso de medicamentos. Ainda, a associação entre diuréticos e betabloqueadores que, segundo Brandão (Coord.), ${ }^{8}$ é uma das associações recomendadas para o controle da HAS, foi classificada no presente estudo como negativa, pelos riscos relacionados à hipotensão e à redução dos batimentos cardíacos. $^{21,22}$

Anlodipino, diuréticos e verapamil podem potencializar o efeito dos betabloqueadores, por potencializar o efeito anti-hipertensivo, sendo a IM com o verapamil uma interação maior, devido ao risco de bradicardia, enquanto os riscos relacionados à IM com anlodipino e diuréticos referem-se a hipotensão e redução do ritmo cardíaco. ${ }^{21}$ Por outro lado, a associação com hidralazina é positiva. Ainda, a fluoxetina pode potencializar o efeito dos betabloqueadores, resultando em aumento do efeito hipotensor e bradicardiazante, o que aumenta o risco de parada cardíaca. ${ }^{18,22}$ Ressalta-se que, mesmo quando um medicamento aumenta o efeito terapêutico de outro, não se pode considerar essa IM positiva, pois pode aumentar os riscos para o usuário. Nesta situação, a dose terapêutica administrada pode ser superior à necessária, sendo imprescindível o monitoramento e, a partir deste, se necessário, o ajuste de doses.

Verapamil pode aumentar a toxicidade de outros fármacos, como betabloqueadores, glibenclamida, sinvastatina e fluoxetina, com atenção especial a associação com betabloqueadores e sinvastatina, com os quais a IM é negativa maior, tendo seus benefícios superados pelos riscos, que incluem bradicardia e rabdomiólise, respectivamente. ${ }^{18,22}$

Anlodipino, quando administrado com anti-hipertensivos como betabloqueadores, IECA e hidroclorotiazida, e ainda com fluoxetina, pode ter seu efeito hipotensor aumentado, ${ }^{20}$ sendo as IM com IECA e hidroclorotiazida positivas. Enquanto a interação com sinvastatina é negativa maior, por potencializar o risco de miopatia pelas estatinas. ${ }^{20}$

Os IECA, outra classe de primeira escolha para o controle da HAS, pode interagir de forma grave com espironolactona, devido ao aumento da toxicidade e risco de hipercalemia. ${ }^{18,21}$ Seu efeito redutor da pressão arterial pode ser aumentado por furosemida, hidroclorotiazida, verapamil e anlodipino, sendo estas IM positivas, enquanto a associação com a metformina pode ocasionar acidose lática e hipercalemia, uma interação negativa. ${ }^{20,21}$

Hidralazina e losartana são os fármacos antihipertensivos com o menor número de IM negativas. Destaca-se que a losartana é eficaz no tratamento da HAS, sendo indicada principalmente na população com alto risco cardiovascular ou comorbidades, proporcionando redução da morbidade e da mortalidade cardiovasculares. Sendo reconhecida como nefro e cardioprotetor para diabéticos, ${ }^{6,8}$ a losartana apresenta-se como uma alternativa quando HAS, DM e dislipidemias são coexistentes. Em relação às IM, se coadministrada com IECA o efeito anti-hipertensivo pode ser aditivo, sendo que a classificação dessa IM varia em moderada $^{20}$ e maior, ${ }^{21}$ por aumentar o risco de efeitos adversos como hipotensão e hipocalemia. Ainda, apresenta IM maior com espironolactona, aumentando o risco de hipercalemia. ${ }^{22}$

Entre os medicamentos antidiabéticos, a classe das sulfoniluréias (glibenclamida e gliclazida) apresenta interação positiva com metformina, sendo a associação de primeira escolha para o controle glicêmico. ${ }^{8}$

Metformina, medicamento indicado para diabéticos obesos, interage com betabloqueadores podendo ocorrer hiperglicemia, hipoglicemia e hipertensão, enquanto enalapril, furosemida e hidroclorotiazida aumentam os risco de ocorrência de acidose lática e hipercalemia. ${ }^{20,21}$

Glibenclamida e gliclazida interagem com betabloqueadores e podem alterar o metabolismo glicêmico causando hiperglicemia, hipoglicemia e hipertensão; e com furosemida e hidroclorotiazida, os quais podem diminuir a tolerância a glicose e causar hiperglicemia. ${ }^{18,20-22}$ Ainda, glibenclamida pode ter seu efeito hipoglicêmico aumentado pelo fenofibrato, fluoxetina, IECA e sinvastatina. ${ }^{20,21}$

Os dois medicamentos com ação hipolipemiante presentes na RENAME ${ }^{17}$ interagem entre si: a concentração sérica de sinvastatina pode ser aumentada pela coadministração de fenofibrato, podendo aumentar o risco de miopatia e rabdomiólise. ${ }^{18,21}$ A classe das estatinas, que inclui a sinvastatina, compreende os medicamentos de primeira escolha para o tratamento das dislipidemias devido à maior evidência na redução da morbimortalidade cardiovascular. ${ }^{8}$ No entanto, verifica-se que quando utilizada em monoterapia, reduz os níveis de colesterol ligado a lipoproteína de densidade baixa (LDL-colesterol) e os riscos cardiovasculares relacionados. ${ }^{8,16}$ Diante disso, é possível inferir que não existem alternativas seguras 
disponíveis na RENAME ${ }^{17}$ para associações entre hipolipemiantes. Destaca-se que quando a associação entre sinvastatina e fenofibrato é imprescindível, é necessário monitorar sinais e sintomas específicos, como miosite, miopatia, rabdomiólise, dor, fraqueza ou rigidez muscular, e a concentração sérica de creatina cinase ${ }^{18}$ Ainda, quando administrada com verapamil e anlodipino a sinvastatina pode ter sua toxicidade aumentada, com risco de miopatia e rabdomiólise, ${ }^{18,20-22}$ enquanto a interação com a hidralazina aumenta o risco de neuropatia periférica. ${ }^{20}$

Fluoxetina, se administrada concomitantemente a anlodipino, betabloqueadores, glibenclamida e verapamil, pode aumentar o efeito farmacológico destes medicamentos. ${ }^{18,22}$ Glibenclamida, por ser um fármaco indutor da coenzima P-450, pode aumentar o metabolismo da fluoxetina ${ }^{22} \mathrm{e}$, ainda, apresenta potencial IM com diuréticos, aumentando os riscos de hiponatremia. ${ }^{20}$

Observou-se que fluoxetina interage com uma classe de primeira escolha dos anti-hipertensivos, os betabloqueadores, podendo causar bradicardia. ${ }^{18}$ Além disso, quando usada no tratamento da depressão, pode promover perda de peso, entretanto, não tem indicação formal para o tratamento da obesidade, pois seu efeito de perda de peso é transitório, não sendo eficiente para o tratamento em longo prazo da obesidade. ${ }^{16}$

Entre os medicamentos presentes na I-DBSM ${ }^{8}$ recomendados para portadores de SM obesos ou com excesso de peso, representados por anfepramona, femproporex, mazindol, sibutramina e orlistat, apenas dois estão liberados no Brasil, sibutramina e orlistat ${ }^{23}$ e nenhum se encontra na RENAME. ${ }^{17}$

\section{DISCUSSÃO}

A falta de consenso sobre a definição de SM implica na utilização de critérios diferentes nos estudos realizados sobre o tema, o que dificulta a comparação entre os resultados dos mesmos. No entanto, independente da definição, sabe-se que a SM é estabelecida por um conjunto de anormalidades metabólicas prevalentes na população, que a condiciona a elevado risco cardiovascular, necessitando de atenção especial para o seu controle, incluindo tratamento medicamentoso adequado. ${ }^{8}$

Inicialmente essa síndrome foi denominada "síndrome X", definição apresentada por Reaven em 1988. ${ }^{24}$ O termo "síndrome metabólica" foi sugerido em 1999 pela OMS, que preconiza como ponto de partida para o seu diagnóstico, a avaliação da resistência à insulina ou de distúrbio no metabolismo da glicose associado a dois ou mais componentes, como aumento da pressão arterial sistêmica, elevados níveis de triglicerídeos no plasma, obesidade central e microalbuminúria. ${ }^{25}$

Estudos baseados na definição do NCEP ATP III ${ }^{9}$ demonstram que a SM está presente principalmente nas faixas etárias mais avançadas. Esse relatório apresenta uma nova definição da SM que não prioriza a presença da resistência a insulina, mas considera que a SM ocorre pela presença de três entre estes cinco fatores: obesidade central, elevado nível de triglicerídeos, reduzido nível de HDL-colesterol, elevada pressão arterial e elevada concentração de glicose no sangue. ${ }^{9}$ Em estudo realizado no Canadá com maiores de 18 anos, a prevalência de SM foi de $19,1 \%$, predominando a faixa etária acima de 60 anos. ${ }^{26}$ Na população americana acima de 20 anos encontrouse prevalência de SM de $34 \%$, e essa porcentagem é representada principalmente por mulheres acima de 60 anos. $^{27}$

Escobedo et al., ${ }^{28}$ ao analisarem a SM em sete grandes cidades da América Latina, verificaram prevalência entre 14 e $27 \%$. No Brasil, em uma revisão sobre o tema, Lopes, ${ }^{29}$ verificou, entre os adultos, variação entre 8,6 e $89 \%$ e, em crianças e adolescentes, entre 0 e $42,4 \%$, indicando que, mesmo sendo prevalente nas faixas etárias avançadas, a SM não é exclusiva de idosos.

Nesse contexto, observa-se que a SM tende a continuar com alta incidência nos próximos anos, além disso, hábitos de vida, como alimentação inadequada e sedentarismo, que predispõem às DCNT que constituem a SM, estão cada vez mais evidentes na sociedade atual. Diante disso, é fundamental a implantação de programas de prevenção e o estabelecimento de esquemas de tratamento seguros. Para a prevenção e tratamento inicial de doenças relacionadas a anormalidades metabólicas são indicadas medidas não farmacológicas, que incluem mudanças dos hábitos de vida, como adoção de alimentação saudável, prática regular de atividade física e combate ao tabagismo, ao consumo de álcool e ao estresse. ${ }^{8}$

Entretanto, pela não adesão aos hábitos saudáveis propostos, ou na eventualidade de os novos hábitos não promoverem as respostas esperadas no controle das doenças, na grande maioria das vezes é necessária a instituição de terapia medicamentosa para o tratamento da SM, que se torna complexa, pois requer o uso concomitante de vários medicamentos. Esta característica eleva o risco de IM, que podem resultar em efeitos adversos ou alterações nos efeitos farmacológicos dos fármacos, expondo o usuário a riscos. ${ }^{22}$ Além disso, existem medicamentos que estão 
mais associados à ocorrência de efeitos indesejados graves quando sua ação farmacológica é alterada, como os de baixo índice terapêutico e os que requerem controle cuidadoso de dose, como no caso de antihipertensivos $\mathrm{e}$ antidiabéticos, ${ }^{12}$ frequentemente utilizados para o tratamento da SM.

Amaral e Perassolo, ${ }^{30}$ ao verificarem as potenciais IM entre anti-hipertensivos e antidiabéticos em 45 participantes de um grupo Hiperdia (Programa Nacional de Assistência Farmacêutica para HAS e DM do Ministério da Saúde) de Parobé, Rio Grande do Sul, encontraram que $87 \%$ dos entrevistados apresentavam alguma IM entre os medicamentos utilizados, com média de 3,7 IM por entrevistado. Santos et al., ${ }^{31}$ ao analisarem as prescrições de 600 hipertensos de Pradópolis, São Paulo, identificaram 1.440 potenciais IM, com média de 2,4 IM por paciente. Locatelli, ${ }^{32}$ em uma Unidade de Internação de Geriatria, verificou 705 potenciais IM entre 155 pacientes, com aproximadamente quatro IM por paciente. Destaca-se que os estudos supracitados identificaram apenas IM negativas, não apresentando as IM positivas.

IM são frequentes e precisam ser constantemente prevenidas. Neste sentido, conhecer as IM potenciais entre os medicamentos indicados para o controle da SM permite evitar as de risco e privilegiar as mais seguras, com menor risco de desencadear efeitos indesejáveis e que viabilizam melhores respostas terapêuticas. Destaca-se que a I-DBSM,${ }^{8}$ que apresenta os medicamentos disponíveis no Brasil que podem ser utilizados para o controle das DCNT que compõem a SM, não discute o uso concomitante desses medicamentos.

Verificou-se através deste estudo que várias são as IM que podem ocorrer quando medicamentos antihipertensivos são associados, algumas positivas, com eficácia comprovada, no entanto, mesmo entre as associações nas quais se buscam uma melhor resposta terapêutica existem riscos relacionados, ao aumento dos efeitos adversos desses medicamentos. Ainda, observaram-se várias IM quando coadministrados com medicamentos antidiabéticos, hipolipemiantes e para a obesidade, sendo fundamental uma assistência individualizada, considerando as doenças que acometem o usuário desses medicamentos e o riscobenefício da associação dos medicamentos.

Nesse contexto, o presente estudo apresenta as potenciais interações a serem evitadas e as alternativas mais seguras, tais como, a utilização de outras classes de anti-hipertensivos, como anlodipino e losartana, que não apresentam IM negativas com antidiabéticos, oportunizando prescrições seguras e racionais.
A ausência no Brasil de medicamentos recomendados para as pessoas com SM e obesidade ou excesso de peso pode resultar em dificuldades de acesso a esses medicamentos, principalmente devido ao custo. No entanto, essa ausência na RENAME pode estar relacionada ao fato que muitos medicamentos para obesidade foram retirados do mercado brasileiro por apresentarem relação risco-benefício desfavorável e efetividade a longo prazo questionável. ${ }^{33} \mathrm{Ainda}$, em relação ao tratamento medicamentoso da obesidade, é importante salientar que dieta e exercício físico são opções terapêuticas de primeira escolha, e que tratamentos farmacológicos devem ser administrados apenas a pacientes que não respondem a mudanças comportamentais. ${ }^{33}$

Ao mesmo tempo em que o presente estudo identificou e discutiu as potenciais IM entre os medicamentos indicados para o controle das DCNT que constituem a SM, buscando apresentar as associações mais seguras disponíveis na RENAME ${ }^{17}$ o que torna seu acesso mais fácil por integrarem a lista básica de medicamentos, apresenta como limitação não identificar os vários esquemas de tratamento prescritos aos portadores de DCNT e a segurança dessas associações, especialmente quando constituem a SM. Também não foram abordadas as manifestações das potenciais IM identificadas.

Destaca-se que a I-DBSM ${ }^{8}$ elenca os medicamentos indicados para o controle da SM, no entanto, não fornece informações relacionadas a IM. Ainda, ao desenvolver o presente estudo observou-se que o FTN, ${ }^{18}$ que contém informações científicas sobre os medicamentos da RENAME, ${ }^{17}$ visando subsidiar profissionais de saúde para a prescrição e dispensação, não apresenta algumas IM encontradas em outras fontes, dificultando a identificação das mesmas pelos profissionais que atuam no Sistema Único de Saúde (SUS).

Considerando que provavelmente grande parte desses medicamentos esteja presente nas Relações Municipais de Medicamentos Essenciais, cabe aos profissionais de saúde, especialmente os envolvidos na programação, seleção e aquisição de medicamentos, bem como prescritores e dispensadores, conhecer, identificar e prevenir IM, e quando a associação dos medicamentos é necessária, a equipe de saúde deve monitorar esse usuário em relação aos possíveis efeitos colaterais resultantes. O farmacêutico tornase fundamental nesse processo, tendo em vista que pode fornecer informações para subsidiar decisões fundamentadas e selecionar uma melhor terapêutica, minimizando riscos ao usuário. Ressalta-se que as IM negativas constituem a maioria das IM identificadas, e 
ocorrem tanto entre medicamentos com a mesma ação farmacológica quanto com ação diferente, destacando a necessidade de uma assistência individualizada aos indivíduos com SM.

A elaboração de protocolos clínicos relacionados ao tratamento da SM, incluindo os medicamentos que podem ser utilizados concomitantemente quando existe mais de uma DCNT e a elaboração de formulários contendo informações sobre as IM negativas mais frequentes, assim como os riscos a elas relacionados, são ferramentas que poderiam auxiliar a equipe multidisciplinar de saúde a identificar as potenciais IM, selecionar medicamentos alternativos, ajustar doses e monitorar o usuário, promovendo um tratamento eficaz e seguro da SM. ${ }^{32}$

Concluindo, o tratamento da SM é complexo por ser necessária a associação de vários medicamentos, o que aumenta os riscos de IM e de efeitos adversos. No presente estudo foi identificado um grande número de medicamentos disponíveis no Brasil para o controle das DCNT que constituem a SM, entretanto uma pequena parte está presente na RENAME e de fácil acesso à população. Identificou-se um grande número de potenciais IM negativas entre esses medicamentos, o que pode reduzir ou potencializar a eficácia dos medicamentos e expor o usuário a riscos.

\section{REFERÊNCIAS}

1. Alves LC, Quinet Leimann BC, López Vasconcelos ME, Sá Carvalho M, Godoi Vasconcelos AG, Oliveira da Fonseca TC, Lebrão ML, Laurenti R. A influência das doenças crônicas na capacidade funcional dos idosos do Município de São Paulo, Brasil. Cad Saude Publica. 2007 Aug;23(8):1924-30.

2. Martins LN, Souza LS, Silva CF, Machado RS, Silva CEF, Vilagra MM, Carvalho CVA, Pereira ABCNG. Prevalência dos Fatores de risco cardiovascular em adultos admitidos na unidade de dor torácica em Vassouras, RJ. Rev Bras Cardiol. 2011;24(5):299-307.

3. Organização Mundial da Saúde. Cuidados inovadores para condições crônicas: componentes estruturais de ação: relatório mundial. Brasília: OMS; 2003.

4. Organización Panamericana de La Salud, Organización Mundial de La Salud. Información y análisis de salud (HSD/HA): situación de salud em las Américas: indicadores básicos 2012. Washington: OPAS/OMS; 2012.

5. Brasil, Ministério da Saúde. Indicadores e dados básicos para a Saúde - IDB 2011 Brasil. Rio de Janeiro: Athalaia; 2011.

6. Nobre F, Editor. VI Diretrizes brasileiras de hipertensão. Revista Brasileira de Hipertensão. 2010;17(1):1-64.

7. Ebrahim S, Montaner D, Lawlor DA. Clustering of risk factors and social class in childhood and adulthood in British women's heart and health study: cross sectional analysis. BMJ. 2004 Apr 10;328(7444):861.

8. Brandão AP, Coordenador. I Diretriz brasileira de diagnóstico e tratamento da síndrome metabólica. Sociedade Brasileira de Cardiologia. Arq Bras Cardiol. 2005;84(supl.1):1-28.

9. Expert Panel on Detection, Evaluation, and Treatment of High Blood Cholesterol in Adults. Executive Summary of The Third Report of The National Cholesterol Education Program (NCEP) Expert Panel on Detection, Evaluation, And Treatment of High Blood Cholesterol In Adults (Adult Treatment Panel III). JAMA. 2001 May 16;285(19):2486-97.

10. Secoli SR. Polifarmácia: interações e reações adversas no uso de medicamentos por idosos. Rev Bras Enferm. 2010;63(1):136-40.

11. Didati AC, Colet C. Interações medicamentosas potenciais em ambiente hospitalar: uma revisão bibliográfica. Rev Cont Saúd. 2010;9(18): 29-36.

12. Hoefler R. Interações Medicamentosas. In: Brasil. Ministério da Saúde. Secretaria de Ciência, Tecnologia e Insumos Estratégicos. Departamento de Assistência Farmacêutica e Insumos Estratégicos. Formulário terapêutico nacional 2008: Rename 2006. Série B. Textos Básicos de Saúde. Brasília: Ministério da Saúde; 2008.

13. Lima REF. Interações medicamentosas potenciais em pacientes de Unidade de Terapia Intensiva de um Hospital Universitário do Ceará [dissertação]. [Ribeirão Preto]: Universidade de São Paulo; 2007. 142 p.

14. Marins N, Editor. Tratamento e Acompanhamento do Diabetes mellitus: diretrizes da Sociedade Brasileira de Diabetes. Rio de Janeiro: Sociedade Brasileira de Diabetes; 2007.

15. Associação Brasileira para o Estudo da Obesidade e da Síndrome Metabólica-ABESO. Diretrizes brasileiras de obesidade. $3^{\mathrm{a}}$ ed. Itapevi/ SP: AC Farmacêutica; 2009.

16. Sposito AC, Editor. IV Diretriz Brasileira Sobre Dislipidemias e Prevenção da Aterosclerose Departamento de Aterosclerose da Sociedade Brasileira de Cardiologia. Arq Bras Cardiol. 2007;88(sup.I):2-19.

17. Brasil. Ministério da Saúde. Secretaria de Ciência, Tecnologia e Insumos Estratégicos. Departamento de Assistência Farmacêutica e Insumos Estratégicos. Relação nacional de medicamentos essenciais: rename. $7^{\mathrm{a}}$ ed. Brasília: Ministério da Saúde; 2010. Serie B. Textos Básicos de Saúde.

18. Brasil. Ministério da Saúde. Secretaria de Ciência, Tecnologia e Insumos Estratégicos. Departamento de Assistência Farmacêutica e Insumos Estratégicos. Formulário terapêutico nacional 2010: rename 2010. 2a ed. Brasília: Ministério da Saúde; 2010. Série B. Textos Básicos de Saúde. 
19. World Health Organization. Collaborating Centre for Drug Statistics Methodology. Anatomical Therapeutic Chemical ATC/DDD Index 2014 [Internet]. Oslo; 2014. [cited 2014 Mar 10]. Available from: http://www.whocc.no/atc_ddd_index/.

20. Drug Information Online. Drug Interactions Checker [Internet]. [cited 2012 Oct 21]. Available from: http://www.drugs.com/drug interactions.php

21. Micromedex Healthcare Series: Interactions. Versão 2.0 [Internet]. [cited 2012 Dec 19]. Available from: http://www-thomsonhc-com. ez45.periodicos.capes.gov.br/micromedex2/librarian

22. Tatro DS. Drug Interaction Fact ${ }^{\mathrm{sTM}}$. Missouri: Wolters; 2006.

23. Brasil. Resolução RDC N $\mathrm{N}^{\mathrm{o}} 52$, de 6 de outubro de 2011. Dispõe sobre a proibição do uso das substâncias anfepramona, femproporex e mazindol, seus sais e isômeros, bem como intermediários e medidas de controle da prescrição e dispensação de medicamentos que contenham a substância sibutramina, seus sais e isômeros, bem como intermediários e dá outras providências. DOU. 2011 Oct. 10;195(1):55.

24. Reaven GM. Banting lecture 1988: role of insulin resistance in human disease. Diabetes. 1988 Dec;37(12):1595-607.

25. World Health Organization. Department of Noncommunicable Disease Surveillance. Definition, Diagnosis and Classification of Diabetes Mellitus and its Complications. Geneva: WHO; 1999.

26. Riediger ND, Clara I. Prevalence of metabolic syndrome in the Canadian adult population. CMAJ. 2011 Oct 18;183(15):E1127-34.

27. Ervin B. Prevalence of metabolic syndrome among adults 20 years of age and over, by sex, age, race and ethnicity, and body mass index: United States, 2003-2006. Natl Health Stat Report. 2009 May 5;(13):1-7.

28. Escobedo J, Schargrodsky H, Champagne B, Silva H, Boissonnet CP, Vinueza R, Torres M, Hernandez R, Wilson E. Prevalence of the Metabolic Syndrome in Latin America and its association with sub-clinical carotid atherosclerosis: the CARMELA cross sectional study. Cardiovasc Diabetol. 2009 Sept 26;8:52.

29. Lopes MJS. Prevalência de Síndrome Metabólica no Brasil: um estudo de revisão [Monografia]. Maringá: Universidade Estadual de Maringá; 2009.

30. Amaral DMD, Perassolo MS. Possíveis interações medicamentosas entre os anti-hipertensivos e antidiabéticos em participantes do Grupo HIPERDIA de Parobé, RS (Uma análise teórica). Rev Ciênc Farm Básica Apl. 2012;33(1):99-105.

31. Santos JC, Faria Junior M, Restini CBA. Potenciais interações medicamentosas identificadas em prescrições a pacientes hipertensos. Rev Bras Clin Med. 2012;10(4):308-17.

32. Locatelli J. Interações medicamentosas em idosos hospitalizados. Einstein. 2007;5(4):343-6.

33. Paumgartten FJR. Tratamento farmacológico da obesidade: a perspectiva da saúde pública. Cad. Saúde Pública. 2011;27(3):404-5. 